\title{
How and Why the Universe Is Natural
}

\author{
Jacques Consiglio $^{1}$ \\ ${ }^{1}$ Labastidette, France \\ Correspondence: Jacques Consiglio, Labastidette, France. E-mail: Jacques.Consiglio@gmail.com
}

Received: April 10, 2014 Accepted: April 23, 2015 Online Published: April 29, 2015

doi:10.5539/apr.v7n3p69 URL: http://dx.doi.org/10.5539/apr.v7n3p69

\begin{abstract}
In previous paper, we showed that the elementary particles mass spectrum can be computed from theory using a resonance equation. The predicted $\mathrm{H}^{0}$ mass agrees with the latest CERN publications. As a result, the $\mathrm{H}^{0}$ acquires its mass exactly in the same geometry as the $\mathrm{Z}^{0}$ and the $\mathrm{W}^{ \pm}$; it is not a "purely scalar boson" and then quadratic divergences are inexistent. In this paper, we find an underlying structure of the field with 19 pure states, where 36 quark states (including color) and 3 or 6 neutrinos states are mixtures, and we show that the CKM and PMNS matrices elements come from the equation and the field structure. We conclude that the field is natural since over the 23 SM parameters, at least 19 are fully constrained by the field structure.
\end{abstract}

Keywords: naturalness, free parameters, CKM matrix, PMNS matrix, Higgs boson, field below

\section{Introduction}

One mystery of the time is the very small mass of the Higgs boson; augmented with quadratic divergences it seems to opens the roads to new physics. But it is also part of a wider mystery, namely the long list of free parameters that feed all viable theories. At present time, none predicts those and the multiverse assumption becomes tempting.

In a non-conventional way, starting with de Broglie's thesis and numerical data, we showed (Consiglio, 2014a, 2014b) that the elementary particles mass spectrum depends on a handful of integral numbers. In this paper, we first review the logic, including the mass equation and the numerical results. Once the link with leptons and quarks masses is understood, it shows that the bosons masses are fully constrained, and that the $\mathrm{H}^{0}$ acquires mass exactly in the same manner as the $W^{ \pm}$and $Z^{0}$; hence it is not a purely scalar boson, and a very simple empirical fit exists with the $\mathrm{W}^{ \pm}$mass and the Weinberg angle. It is then a "usual" vector boson that mediates some unknown exchanges, but also suggests a natural candidate to mediate gravitation in an extended electroweak theory.

Next, we picture the field as a whole and reduce it to 19 fundamental objects plus 42 mixed states (quarks and neutrinos); we find that 4 of the 19 objects (corresponding to 4 quarks masses) mix to all quarks and neutrinos. For this, we show that those masses are in close logical and numerical relations with the Cabibbo-Kobayashi-Maskawa (CKM) and Pontecorvo-Maki-Nakagawa-Sakata (PMNS) matrices, and that only the $u$ and $d$ quarks are real mixes. Neutrinos are also shown to be mixes of the same objects. Here, a geometrical or dimensional inversion appears which is reminiscent of the see-saw mechanism.

We conclude that the universe is natural because with 19 coherent standard model (SM) parameters, out of 23, we have largely exceeded the critical point at which the evidences cannot be so consistent in logic and precision without approaching some underlying truth.

\section{Elementary Particles Mass}

\subsection{Deriving a Mass Equation}

The logic is inspired of the Feynman-Wheeler absorber theory (Wheeler \& Feynman, 1945, 1949) and we assume that the wave is the physical exchange at the origin of mass; but energy exchange is momentum, and it gives a pressure field that "cages" the particle charges and self-energy. The Cramer interpretation of quantum mechanics (Cramer, 1986) is complimentary, and we assume that the self-energy is the Cramer wave - which here is permanent. The idea is similar to the Poincaré stress (Poincaré, 1903) though not identical as we split the particle.

The relation $E=h v$ as first used in de Broglie (1924) for massive particles suggests some form of resonance and the standard theory heavily uses the concept, including widths. Moreover, the Compton wavelength $\lambda_{C}=h / m c$ when compared to the de Broglie wavelength $\lambda_{D}=h / m v$ gives a form of symmetry where the light-speed singularity has the role of a mirror that directly applies to velocity (de Broglie's $\mathrm{Vv}=\mathrm{c}^{2}$ ). 
It is minimal to assume that all resonances come from the same fundamental of length 1 (a natural unit). In the one dimensional case, the pressure is a simple force, and resonance implies an integral number $N$ such that we have:

$$
\mathrm{m}=\mathrm{XN}+\mu
$$

where $m$ is the particle mass, and $X$ is a natural constant. At this point, this is just a vibrating string. The coefficient $\mu$ represents a massless self-energy that necessarily propagates, and it implies a double resonance. Hence the actual resonance number is a product $N P$, with $P$ an integral number, and we get:

$$
\mathrm{m}=\mathrm{XNP}+\mu
$$

Caging a massless particle requires symmetry, a repelling force that applies to the particle self-energy and to the pressure field as well; precisely to the first resonance wall $(1 / N P)$ and to the self-energy $\mu$. There must be a residual distance $d \neq 0$ between the first resonance wall and the current $\mu$ at which the force applies. It gives:

$$
\mathrm{m}=\mu+\mathrm{X}\left(\mathrm{d}+(\mathrm{NP})^{-1}\right)^{-1}
$$

Now the distance $d$ also depends on 1/NP because energy comes from the distance $(d+1 / N P)$ and it is equivalent to a potential; actually two potentials that sum or subtract, one is $1 / N P$ and the other is $d$. A potential is quantized; $1 / N P$ is already quantized (in a reverse manner), and then $d$ is also quantized. Then we use $d=K D$, with $K$ an integral number and $D$ a length. In three dimensions, we get a cube:

$$
\mathrm{m}=\mu+\frac{\mathrm{X}}{(1 / \mathrm{NP}+\mathrm{KD})^{3}}
$$

Essentially, this is thermodynamics; $P V=k_{B} T$ (not the same $\mathrm{P}$ as in (1)) with a single molecule $\mu$ in a volume $V=$ $(d+1 / N P)^{3}$, where $k_{B} T=$ constant $=\mu c^{2}$, and the rest of the particle energy is interaction - the Feynman-Wheeler exchanges - while $\mu$ is the Cramer wave. (The point is discussed briefly in addendum).

Now contrary to the one-dimensional problem, we have more degrees of freedom and the resonance paths associated to $N$ and $P$ can have different geometries:

- Case 1: A double radial resonance. It is compatible with $N=P$ and can trivially be identified to $\mathrm{U}(1)$ and (to some extent) to the Poincaré stress in which case we should have $\mathrm{KD}>0, \mathrm{~K}$ increasing with mass.

- Case 2: A double circular resonance: It is also compatible with $N=P$ and the forces at work in the resonance correspond to a symmetry group that inverts rotation axis; hence it must be identified to $\mathrm{SU}(2)$. Moreover, in this case, we should find $X \rightarrow X / n \pi$; this is because, compared to the first case, even though the resonance is circular the pressure is still applied to its geometrical center.

- Case 3: A mixed resonance implies $N \neq P$ with a geometrical constraint between $\pi, N$ and $P$ since we must have a phase lock between the two paths; hence we should get approximate equalities like:

$$
N P \pi \approx \text { an integral number }
$$

The last known symmetry is $\mathrm{SU}(3)$ and quarks should correspond to this type of resonance. If $\mathrm{D}$ is related to the strong force, we should have $\mathrm{KD}<0$, ideally constant (if related to asymptotic freedom).

Hence the resonance concept used implies three symmetries that we must identify to the known ones; but here in the best case, the symmetries are the resonance geometry and do not need to originate in separate fields. The equation has 6 degrees of freedom and this is sufficient to assess the leptons and the bosons masses separately, but not necessarily the quarks; one of the main tasks in computing the mass spectrum is to reduce this number.

\subsection{Particles Resonances}

\subsubsection{Leptons}

The Table 1 shows charged leptons resonances. We find very small numbers $(\mathrm{N}=\mathrm{P}$, and $\mathrm{K})$ and a regular pattern.

Table 1. Electrons, muon, tau. $(*) \mathrm{MeV} / \mathrm{c}^{2}$

\begin{tabular}{llllll}
\hline Particle & $\mathrm{P}$ & $\mathrm{N}$ & $\mathrm{K}$ & Computed $(*)$ & Measured $(*)$ \\
\hline Electron & 2 & 2 & 2 & 0.5109989280 & $0.510998928(11)$ \\
Muon & 5 & 5 & 3 & 105.65837150 & $105.6583715(35)$ \\
Tau & 9 & 9 & 5 & 1776.840 & $1776.82(16)$ \\
\hline
\end{tabular}


Parameters are hereafter and will be used as constraints for the other particles groups:

$$
\mu=241.676611 \mathrm{eV} ; D_{\mathrm{e}}=0.00085322189 \mathrm{~L} ; \mathrm{X}=8.145121041623 \mathrm{KeV} \mathrm{L}^{3}
$$

( $L=$ lengths natural unit.)

Using $\alpha$, the fine structure constant, we define a new constant that will be later used:

$$
\mathrm{A}_{\mathrm{S}}=\mathrm{D}_{\mathrm{e}} / \alpha=0.1169221145
$$

The name $A_{S}$ is chosen for its value is reminiscent of the strong force coupling and the position of $\mathrm{D}$ in the equation is also reminiscent of the strong force. It is easy to guess:

$$
\alpha_{\mathrm{S}(\mathrm{MZ})}=\mathrm{A}_{\mathrm{S}}(1+\alpha \sqrt{ } 3)=0.118399
$$

where the term $\sqrt{ } 3$ is geometrical; $\alpha$ is the fine structure constant which, together with the interaction scale MZ will be discussed in the following sections.

\subsubsection{Quarks}

Table 2 shows the resonance numbers we found for quarks, where masses are coherently expressed in the natural scheme. A regular pattern is obvious. One parameter is changed to compute this table:

$$
\mathrm{D}_{\mathrm{q}}=\mathrm{D}_{\mathrm{e}}(1+\alpha)
$$

It shows a connection between $\mathrm{D}_{\mathrm{e}}$ and $\alpha_{\mathrm{S}}$ (without which the top quark mass is computed around $167 \mathrm{GeV}$ ). The constancy of $\mathrm{K}=-6$ is reminiscent of asymptotic freedom.

Table 2. Quarks resonances. $\left({ }^{*}\right) \mathrm{MeV} / \mathrm{c}^{2}$, Top $=$ direct measurement $-\left({ }^{1}\right)$ CMS (2014b). See also: The ATLAS, CDF, CMS and D0 Collaborations (2013).

\begin{tabular}{lllllll}
\hline Particle & Charge & $\mathrm{P}$ & $\mathrm{N}$ & $\mathrm{K}$ & Computed $\left(^{*}\right)$ & Estimate $\left(^{*}\right)$ \\
\hline Up & $2 / 3$ & 3 & 2 & -6 & 1.93 & $1.7-3.1$ \\
Down & $1 / 3$ & 3 & $19 / 7$ & -6 & 5.00 & $4.1-5.7$ \\
Strange & $1 / 3$ & 3 & 7 & -6 & 106.4 & $80-130$ \\
Charm & $2 / 3$ & 3 & 14 & -6 & 1,255 & $1180-1340$ \\
Bottom & $1 / 3$ & 3 & 19 & -6 & 4,285 & $4130-4370$ \\
Top & $2 / 3$ & 3 & 38 & -6 & 172,380 & $172,040 \pm 190 \pm 750\left(^{1}\right)$ \\
\hline
\end{tabular}

We get $N \neq P$ in Table 2 , as expected for quarks. It is trivial to verify the expected approximate relations with $N P \pi$, from which it is evident that the integral numbers in Table 2 are not random but geometrical.

For the down quark we get a fraction $\mathrm{N}=19 / 7$ which is not acceptable. The point will be discussed later with the CKM matrix and we shall see that it comes from mixing. Still we notice that all variable numbers come from 2, 7 , and 19 and the ratio 2 within generations is the ratio of electric charge; it suggests a unique geometry.

\subsubsection{Massive Bosons}

The remaining resonance mode is doubly circular, then compatible with $\mathrm{N}=\mathrm{P}$. Using the SM we must naturally assume that the $\mathrm{W}^{ \pm}, \mathrm{Z}^{0}$ and $\mathrm{H}^{0}$ acquire their masses from the same potential; using (1), it should correspond to the same resonance and coefficients, that is $\mathrm{N}=\mathrm{P}=$ constant, and only $\mathrm{K}$ varies.

As mentioned before, an important aspect in this resonance mode is that equation (1) is modified:

$$
\mathrm{m}=\mu+\frac{\mathrm{X}}{\mathrm{k} \pi(1 / \mathrm{NP}+\mathrm{KD})^{3}}
$$

The factor $\pi$ at the denominator is needed since the resonances is supposed circular, but we do not find a good fit; we need a factor $k \approx 1$, another degree of freedom that will be removed.

The next reasoning is long (for details, refer to Consiglio, 2014b), and it shows that $D_{b}$ is an interaction term on which $k$ depends thru the following geometrical equation that connects the resonant "slots" in two hyper-spheres:

$$
\mathrm{k}^{3} \pi / 144=266 \mathrm{D}_{\mathrm{b}}(\pi / \mathrm{k})^{1 / 3}
$$


The interaction is decrypted in the same reasoning (and paper) and gives distinct terms in $\mathrm{D}_{\mathrm{b}}$ for the $\mathrm{W}^{ \pm}, \mathrm{Z}^{0}$, or $\mathrm{H}^{0}$ :

$$
\begin{aligned}
\mathrm{D}_{\mathrm{WZ}} & =\alpha^{2}\left(1 /\left(1+\alpha^{2}\right)+\mathrm{A}_{\mathrm{S}} / 2\left(1-\alpha^{2}\right)-\mathrm{A}_{\mathrm{S}}^{2} / 6\left(1+\alpha^{2}\right)\right)=5.6240490410^{-5} \\
\mathrm{D}_{\mathrm{H}} & =\alpha^{2}\left(1 /\left(1+\alpha^{2}\right)+\mathrm{A}_{\mathrm{S}} / 2\left(1-\alpha^{2}\right)-\mathrm{A}_{\mathrm{S}}^{2} /\left(1+\alpha^{2}\right)\right)=5.5633866410^{-5}
\end{aligned}
$$

Note that only the last term is different by a factor 6 between those expressions. Note also that the bosons widths are computed (Consiglio, 2014b, 2015) from the resonance geometry using the same difference.

Then the equation (4) gives the coefficients $k$ with which the bosons masses are computed:

$$
\mathrm{k}_{\mathrm{WZ}}=1.00128565 ; \mathrm{k}_{\mathrm{H}}=0.998033312
$$

It gives Table 3, where the coefficient $\mathrm{X}$ is unchanged (masses in natural scheme).

Table 3. Predicted Bosons Masses $\left({ }^{*}\right) \mathrm{MeV} / \mathrm{c}^{2} .\left(^{2}\right)$ ATLAS-CMS (2015); see also ATLAS (2014), CMS (2014a)

\begin{tabular}{llllllll}
\hline Particle & $\mathrm{P}$ & $\mathrm{N}$ & $\mathrm{K}$ & $\mathrm{NP}$ & Computed $\left(^{*}\right)$ & Measured $\left(^{*}\right)$ & SM Prediction $\left(^{*}\right)$ \\
\hline $\mathrm{W}^{ \pm}$ & 12 & 12 & -2 & 144 & $80,384.859$ & $80,385 \pm 15$ & $80,363 \pm 20$ \\
$\mathrm{Z}^{0}$ & 12 & 12 & -7 & 144 & $91,187.560$ & $91,187.6 \pm 2.1$ & $91,187.4 \pm 2.1$ \\
$\mathrm{H}^{0}$ & 12 & 12 & -19 & 144 & $125,206.552$ & $125,090 \pm 240^{(2)}$ & None \\
\hline
\end{tabular}

\subsection{Remarks}

Finally, taking into account the reasoning to $(4-5-6)$, and understanding that the bosons $\mathrm{N}$ and $\mathrm{P}(=12=19-7)$ and the shift $\mathrm{K}(=-2,-7,-19)$ address the same geometry as quarks, there is no remaining degree of freedom in the calculus of Table 3 ( $\mathrm{X}$ and $\mathrm{A}_{\mathrm{S}}$ are computed from leptons, quarks give the other interaction term $\alpha$, and the factor 6 in (5.2) comes from the difference in compositeness between bosons). Hence the theory relates to a single field "below" and it is fundamental that all results are based on charge currents, making neutral currents composite.

\section{What is the $\mathbf{H}^{0}$ ?}

\subsection{Scary Coincidences}

The results in section 2 show that the $\mathrm{H}^{0}$ gets its mass from the same geometry as the other massive bosons, which is not anticipated by the standard theory. It is neutral, like the $\mathrm{Z}^{0}$, and then its mass should be related to the $\mathrm{W}^{ \pm}$mass and the Weinberg angle $\theta_{\mathrm{W}}$. Using the bosons masses in Table 3 (or experimental data) we notice:

$$
\cos \left(\theta_{\mathrm{W}}\right)=\frac{\mathrm{M}_{\mathrm{W}}}{\mathrm{M}_{\mathrm{Z}}} \rightarrow \theta_{\mathrm{W}}=0.4916965 \mathrm{rad} \approx \frac{1}{2} \mathrm{rad}
$$

A similar relation using $2 \theta_{\mathrm{W}}$ for the Higgs would give $\mathrm{M}_{\mathrm{H}} \approx 100 \mathrm{GeV}$ which we know is wrong. Then, paralleling, we define an angle $\theta_{\mathrm{H}}$ as follows:

$$
\tan \left(\theta_{\mathrm{H}}\right)=\frac{\mathrm{M}_{\mathrm{H}}}{\mathrm{M}_{\mathrm{W}}} \rightarrow \theta_{\mathrm{H}}=1.0000533 \mathrm{rad} \approx 1 \mathrm{rad}
$$

Here we begin to guess that the electroweak theory is incomplete; representing those angles on the bosons plane gives Figure 1 (which is outlaw in electroweak theory) and suggests that the Higgs is a doubled $Z^{0}$ with opposite rotation and currents (like the $\mathrm{W}^{+}$versus $\mathrm{W}^{-}$).

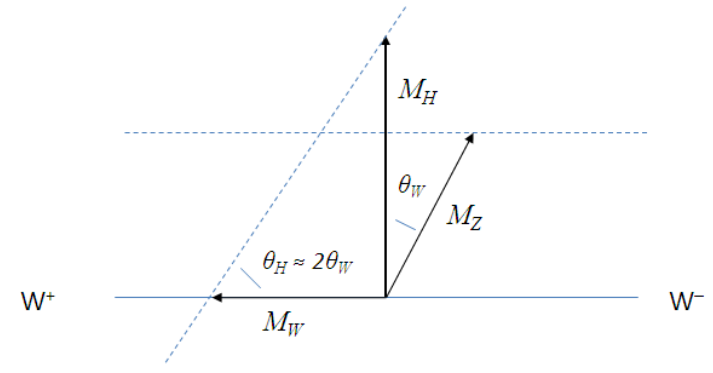

Figure 1: Representing the $\mathrm{H}^{0}$ mass on the bosons plane 
In electroweak parlance, using (9.1) we have:

$$
\left[\begin{array}{c}
\gamma \\
Z^{0}
\end{array}\right] \approx\left[\begin{array}{cc}
\cos \left(\frac{1}{2}\right) & \sin \left(\frac{1}{2}\right) \\
-\sin \left(\frac{1}{2}\right) & \cos \left(\frac{1}{2}\right)
\end{array}\right]\left[\begin{array}{c}
\mathrm{B}^{0} \\
\mathrm{~W}^{0}
\end{array}\right]
$$

Using (9.2) and trigonometry, we get the sum of ratios of the $\mathrm{B}^{0}$ and $\mathrm{W}^{0}$ components of the $\gamma$ and $\mathrm{Z}^{0}$ respectively, and following (10) the probable signature of an incomplete theory is difficult to ignore:

$$
\frac{2 \mathrm{M}_{\mathrm{W}}}{\mathrm{M}_{\mathrm{H}}} \approx \cot \left(\frac{1}{2}\right)-\tan \left(\frac{1}{2}\right)
$$

But those equalities are approximate; then we better write $(9.1-9.2)$ in a manner that addresses the figure, and we estimate angular deviations terms with respect to $1 / 2$ and 1 radian (still using Table 3):

$$
\begin{gathered}
\mathrm{M}_{\mathrm{W}}=\mathrm{M}_{\mathrm{Z}} \times \cos \left(\frac{1}{2}-\alpha-6 \pi \alpha^{2}\right) \\
\mathrm{M}_{\mathrm{H}}=\mathrm{M}_{\mathrm{W}} \times \tan \left(1+\alpha^{2}\left(1-\alpha-3 \pi \alpha^{2}\right)\right)
\end{gathered}
$$

Precision is now $2.610^{-8}$ in (12.1) and $1.610^{-8}$ in (12.2) with respect to the masses computed in Table 3. Hence the coincidence is also evident in the deviation terms.

Using the $\mathrm{H}^{0}$ mass and a "separate" scalar field, the SM implies fine tuning from which the universe does not make sense. Here we find a single field "below" and we guess that the standard theory is incomplete, because it does not gives sense to experimental data.

\subsection{Consequences}

The relatively small mass of the $\mathrm{H}^{0}$ can be seen consistent in two manners, firstly from the resonances (which also explain why the angles are not exactly $1 / 2$ and 1 radian), and secondly from the coincidences in $(9-12)$ as it suggests an incomplete theory. It is an electroweak boson that acquires its mass in the same manner as the others; it shows that all bosons masses are the same consequences of symmetry breaking - which, for the Higgs, is in contradiction with the SM where it is the boson of a separate field. It first eliminates quadratic divergences; since the $\mathrm{H}^{0}$ is not the true "native" boson of a scalar field, those contributions to its mass are irrelevant - in principle, and independently of its decay widths.

But then the $\mathrm{H}^{0}$ is a "usual" vector boson; what force does it mediate? From $(9-11)$ and also using the toy model depicted in the next section, it looks like two opposite $Z^{0}$. It is then a natural candidate to mediate the absorber process that includes an advanced and a retarded wave. But the mediated particle may also have long range effects and the only missing force in the SM is gravitation; but it implies a non-standard (non-bosonic) graviton. Hence the reason why it couples in mass, if experimentally confirmed, is not trivial at all; it might even be double. It leads to conjecture that the long-range gravitational force is residual as it may explain the difference in strengths between gravitation and the weak force. Now let us go back to the main subject.

\section{A Toy Model and a Map}

Before discussing the other SM parameters we must, as a minimum, picture the field in a coherent manner.

\subsection{Modeling Currents}

In our views, the electroweak theory cannot hold in its present state because it is based on the existence of neutral currents: The terms in (5) mean that all fundamental currents are charged and implies definite quanta. It leads to the toy model presented hereafter which was first studied (Consiglio, 2014b) for massive particles.

We first represent currents; they have a sign ( + and - ) and a direction:

- Vertical arrows $\uparrow, \downarrow$ represent time-currents, up and down respectively (fractional electric charges);

- Oblique arrows $\lambda, K$ are space currents, a-priori on the light cone, but causal and anti-causal.

We must now set a-priori rules for assembling currents. They are:

- R1: Time-currents can assemble in all possible ways that give known massive particles.

- R2: Mass and wave emerge from the interaction of space and time-currents.

- R3: Neutral currents are composite.

Implicitly, the apparent charge depends on the current time-direction (like in Dirac's hole theory). A negative electric charge is given by $\uparrow^{-}$for $-2 / 3$ or by $\downarrow_{+}$for $-1 / 3$. (Note the + and - as upper and lower indices.) 
Massive particles are depicted as follows (invert signs for antiparticles where needed):

- Quarks: $\mathrm{t}^{+}:\left[\uparrow^{+} \downarrow_{-} \uparrow^{-} \downarrow_{+} \uparrow^{+}\right] ; \mathrm{b}^{-}:\left[\downarrow_{+} \uparrow^{-} \uparrow^{+}\right] ; \mathrm{c}^{+}:\left[\uparrow^{+} \downarrow_{-} \downarrow_{+}\right] ; \mathrm{s}^{-}:\left[\downarrow_{+}\right]$(u and d are supposed mixes).

- Leptons: $\mathrm{e}^{-}:\left[\uparrow^{-} \downarrow_{+}\right] ; \mu^{-}:\left[\uparrow^{-} \downarrow_{+} \uparrow^{-} \uparrow^{+}\right] ; \tau^{-}:\left[\uparrow^{-} \downarrow_{+} \downarrow_{-} \downarrow_{+}\right]$.

- Bosons: $Z^{0}$ can be: $\left[\uparrow^{+} \uparrow^{-}\right]$or $\left[\downarrow_{+} \downarrow_{-}\right] ; \mathrm{W}^{ \pm}:\left[\uparrow^{+} \downarrow_{-}\right]$and $\left[\uparrow^{-} \downarrow_{+}\right] ; \mathrm{H}^{0}:\left[\uparrow^{+} \uparrow^{-} \downarrow_{+} \downarrow_{-}\right]$.

Note that the $\mathrm{H}^{0}$ is depicted simultaneously as a double $\mathrm{Z}^{0}$ and the sum of a $\mathrm{W}^{+}$and $\mathrm{W}^{-}$. It is then coherent with the Figure 1 and the coincidence in (11) and (12.2) simultaneously.

Some important properties of this model are coherent (Consiglio, 2014b), in particular for quarks and leptons decays, its impact on the equations (5), and to compute the bosons widths; the leptons model was used (Consiglio, $2015)$ to compute their magnetic moment anomaly. So even as a simplified view it should be taken seriously.

Massless particles are depicted as follow (also presented before but modified here to for the "absorber" particle).

- Photon: $\left[\nearrow^{+} K^{+}\right]$, or $\left[\nearrow^{-} K^{-}\right]$(will not be used).

- Neutrino: $\left[\nearrow^{+} \nearrow^{-}\right]$, or $\left[\boldsymbol{K}^{+} \boldsymbol{\swarrow}^{-}\right]$.

- Absorber: $\left[\boldsymbol{\nearrow}^{+} \boldsymbol{\lambda}^{-} \boldsymbol{K}^{+} \boldsymbol{K}^{-}\right]$.

- Color basis, the triplet $\left\{\left[\nearrow^{+}\right],\left[{K^{-}}^{-}\right],\left[\nearrow^{-} K^{+}\right]\right\}$fits with the arithmetic (the demonstration is trivial since $\left[\nearrow^{+} \boldsymbol{\swarrow}^{+}\right]$and $\left[\boldsymbol{\nearrow}^{-} \boldsymbol{\swarrow}^{-}\right]$are color neutral).

\subsection{Picturing the Field Structure}

It can be shown easily that all resonance numbers come from 2, 3, 7, and 19 as sums and products. It was shown (Consiglio, 2015) that the number 37 is also part of the game. Then those numbers address the fundamental field structure: 7, 19, and 37 are centered hexagonal numbers and 61 is the next one - it is also the full SM particles count, but we find neither 37 nor 61 in the resonances. Then in Figure 2, we picture the core field (19 fundamental states) from which we assume that $42(=61-19)$ SM states are mixes. The hexagonal ring structure gives the resonance numbers, and the proposed organization is based on charge continuity and resonance "sectors" (brackets on the figure) where the connection is trivial. One first puts leptons and bosons around the symmetry of electricity; then we add 4 objects (T, B, C, S) which complement the external layer (named, on purpose, after four SM flavor numbers usually denoted T, B', C, S). The 42 other SM states constitute the external layers (rings 37 and 61); they are assumed mixed states of (T, B, C, S), which resonances are the same as four quarks (t, b, c, s). This assumption is shown valid in the next sections as we find direct relations between the four quarks resonances and the CKM and PMNS matrices.
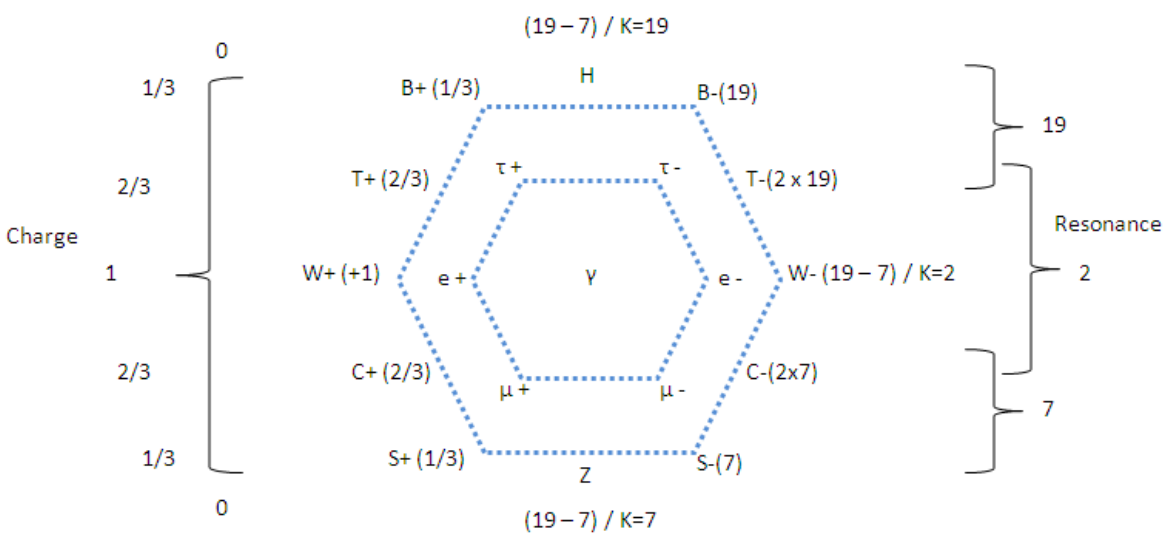

Figure 2. Core field structure, from charge (left half) and resonance (right half)

\section{Other Objects and SM Parameters}

\subsection{The Fine Structure Constant}

The fine structure constant was found to be a pseudo-norm in leptons resonances. The reasoning is long and will not be repeated here. In short, understanding how to compute the leptons magnetic moment anomaly from Table 1 and special relativity leads to guess a second view on the leptons resonances obeying the same equation and a mass $\mu^{\prime} \approx \mu \pi / 2 \approx 380 \mathrm{eV}$; best fit around this value gives Table 4 . 
Table 4. Second view on leptons resonance. $(*) \mathrm{MeV} / \mathrm{c}^{2}$

\begin{tabular}{llllll}
\hline Particle & $\mathrm{P}^{\prime}$ & $\mathrm{N}^{\prime}$ & $\mathrm{K}^{\prime}$ & Computed $(*)$ & Measured $(*)$ \\
\hline Electron & 2 & 2 & 2 & 0.5109989280 & $0.510998928(11)$ \\
Muon & 3 & 8 & 3 & 105.65837150 & $105.6583715(35)$ \\
Tau & 4 & 16 & 4 & 1776.840 & $1776.82(16)$ \\
\hline
\end{tabular}

Parameters are quite different:

$$
\mu^{\prime}=385.674928957 \mathrm{eV} / \mathrm{c}^{2} ; \mathrm{D}^{\prime}=0.0002255984538 ; \mathrm{X}^{\prime}=8.02160767375101 \mathrm{KeV} / \mathrm{c}^{2}
$$

Here it is interesting to look at all distinct integral $\mathrm{N}$ and $\mathrm{P}$ in Tables 1, 2, 3, and 4 as their sum gives 137 - a well-known number which here is just the sum of all resonances. Then 137 as a prime number cannot split as a product, but it effectively splits as a sum. We interpret this number as directly related to the fundamental currents or currents ratio.

Now (still in short) since in Table 4 all N's are harmonics, and since we find $N^{\prime}(\tau)=N^{\prime}(e) N^{\prime}(\mu)$ and $P^{\prime}=K^{\prime}$, we have a unique currents path; together with the fundamental current or ratio 137 it gives a pseudo-norm which is the fine structure constant:

$$
\alpha^{-1}=\sqrt{137^{2}+\pi^{2}-\left(\frac{1}{137}\right)\left(\frac{1}{2}+\frac{1}{8}\right)} \rightarrow \alpha=72973525697.9 \times 10^{-13}
$$

Where CODATA gives:

$$
\alpha=72973525698(24) \times 10^{-13}
$$

Hence $\alpha$ is right there in the geometry of leptons resonance. At this point, it looks like we have "decoded" the electroweak sector parameters since all (in their standard expressions and using the SM) can be computed from the weak bosons masses and the fine structure constant, including the Higgs field v.e.v.

\subsection{The Cabibbo-Kobayashi-Maskawa (CKM) Matrix}

Now let us discuss the main missing group of quarks parameters; that is the CKM matrix in Table 5.

Table 5. The Cabibbo-Kobayashi-Maskawa (CKM) matrix (Particle Data Group, 2014).

\begin{tabular}{rll}
$V u d=0.97427(14)$ & $V u s=0.22536(61)$ & $V u b=0.00355(15)$ \\
$V c d=0.22522(61)$ & $V c s=0.97343(15)$ & $V c b=0.0414(12)$ \\
$V t d=0.00886(32)$ & $V t s=0.0405(11)$ & $V t b=0.99914(5)$ \\
\hline
\end{tabular}

(Uncertainty is minimized, taking the smallest value when non-symmetrical).

In the equation (1) the mass of a quark $q$ depends on a radius $R_{q}=\left(1 / 3 N-6 D_{q}\right)$, which actually defines a potential from a single variable $N$. Hence the quarks decay probabilities should depend on those radiuses or potentials. The second aspect is that between potential wells, we must have different hills which depend on the field structure. Last, using the field map, the quarks field is defined by four objects. Hence widths depend on the $R_{q}$, on some mixing, and simultaneously some numbers related to the field geometry. This is many degrees of freedom, but fortunately the matrix is not quite complex and the problem is limited by the quarks resonances. Looking at Table 3 we remark that the $d$ and $u$ naturally come as ratios from which we assume the form of the mixing:

$$
\begin{gathered}
\mathrm{N}(\mathrm{u})=2=\mathrm{N}(\mathrm{t}) / \mathrm{N}(\mathrm{b})=\mathrm{N}(\mathrm{c}) / \mathrm{N}(\mathrm{s}) \\
\mathrm{N}(\mathrm{d})=19 / 7=\mathrm{N}(\mathrm{t}) / \mathrm{N}(\mathrm{c})=\mathrm{N}(\mathrm{b}) / \mathrm{N}(\mathrm{s})
\end{gathered}
$$

where $\mathrm{N}(\mathrm{q})$ denotes the resonance number $\mathrm{N}$ of the quark q. Now the presence of a fraction (19/7) for the down is not a problem but rather insightful, and $\mathrm{N}(\mathrm{u})=2$ as well since it is simultaneously a ratio of resonances and the ratio of electric charges within the $2^{\text {nd }}$ and $3^{\text {rd }}$ generations. Hence with respect to the field map and (16), it is legitimate to assume that only the $u$ and $d$ are mixes. 


\subsubsection{Computing Coefficients}

Then let start with the top quark decays as it should only depend on field numbers and on the radiuses $R_{d}, R_{s}, R_{b}$. We define a function $f t$ which defines squared ratios with respect to the sum of all potentials, it is:

$$
\mathrm{ft}(\mathrm{q})=\mathrm{R}_{\mathrm{q}}^{2} /\left(\mathrm{R}_{\mathrm{d}}+\mathrm{R}_{\mathrm{s}}+\mathrm{R}_{\mathrm{b}}\right)^{2}
$$

Now the numerical coincidence with the decay widths is easy to find (the numerical results are in Table 6):

$$
\begin{gathered}
\mathrm{Vtb}^{2}=1-\mathrm{ft}(\mathrm{b}) / 3 \\
\mathrm{Vts}^{2}=\mathrm{ft}(\mathrm{s}) / 37 \\
\mathrm{Vtd}^{2}=\mathrm{ft}(\mathrm{d}) \times \pi / 137^{2}
\end{gathered}
$$

where, comparing (17) to the central values in Table 5, the ratio "Error in (17)/Standard Uncertainty" is lesser than $310^{-2}$ for $V t b$ and $V t s$, and $10^{-1}$ for $V t d$. The second heaviest quark is the $b$, for which we use a similar function $f b$ that does not takes the $t$ into account as we want to compute only two points in the right-hand column of the matrix:

$$
\mathrm{fb}(\mathrm{q})=\mathrm{R}_{\mathrm{q}}^{2} /\left(\mathrm{R}_{\mathrm{u}}+\mathrm{R}_{\mathrm{c}}\right)^{2}
$$

From which we find:

$$
\begin{gathered}
\mathrm{Vub}^{2}=\mathrm{fb}(\mathrm{u}) /\left(\pi \times 137^{2}\right) \\
\mathrm{Vcb}^{2}=\mathrm{fb}(\mathrm{c}) / 2 \pi
\end{gathered}
$$

The precision obtained for $V c b$ is in the same range as for the top quark decays, but it is barely in the standard uncertainty range for $V u b$. Together with the error in $V t d$, this is expected since only the $u$ and $d$ are assumed mixed states. Here we need empirical corrections for those coefficients; the following holds:

$$
\begin{aligned}
& \mathrm{Vtd}^{2}=\mathrm{ft}(\mathrm{d}) \times\left(\pi / 137^{2}\right) \times(1+1 / 137) \\
& \mathrm{Vub}^{2}=\mathrm{fb}(\mathrm{u}) \times(1-1 / 14) /\left(\pi \times 137^{2}\right)
\end{aligned}
$$

Now back to the mix in (16); except for some ad hoc coefficient, a ratio of resonances is a product of lengths, and then in order to compute coefficients we just need symmetric expressions that sum lengths products, and the mixing form in (16) leaves no choice. We define in (20) two corresponding quantities:

$$
\begin{aligned}
& A=\left(R_{b} R_{c}\right)+\left(R_{t} R_{s}\right) \\
& B=\left(R_{t} R_{b}\right)+\left(R_{c} R_{s}\right)
\end{aligned}
$$

Now compute the following expression, where the ad hoc coefficient cancels:

$$
\tan ^{-1}((\mathrm{~A} / \mathrm{B}) / 2)=12.94^{\circ}
$$

The Cabibbo angle is $\theta_{C} \approx 13.02^{\circ}$, the difference with $(20.3)$ is -0.08 degrees $(\approx 0.6 \%)$. The factor 2 can be explained if the physical coupling point is halfway thru the mixes $A$ and $B$. Last we find empirical corrections to (20.3) which are mixes of the corrections in (19) in form and coefficients:

$$
\begin{gathered}
\tan ^{-1}((\mathrm{~A} / 2 \mathrm{~B}) \times(1+(1 / 137) \times(1-1 / 14)))=13.025^{\circ}=\cos ^{-1}(\mathrm{Vud})=\sin ^{-1}(\mathrm{Vus}) \\
\tan ^{-1}((\mathrm{~A} / 2 \mathrm{~B}) \times(1+(1 / 137) \times(1-1 / 7)))=13.019^{\circ}=\sin ^{-1}(\mathrm{Vcd}) \\
\tan ^{-1}((\mathrm{~A} / 2 \mathrm{~B}) \times(1+(3 / 137) \times(1+1 / 14)))=13.234^{\circ}=\sin ^{-1}(\mathrm{Vcs})
\end{gathered}
$$

Using the angles in $(21)$ and $(17.2,17.3,18.2,19.1,19.2)$ gives precision as shown in Table 6 , where the numbers in parenthesis are the error with respect to the central value in Table 5.

Table 6. Resulting CKM matrix

\begin{tabular}{lll}
\hline$V u d=0.97427(0)$ & $V u s=0.22538(+2)$ & $V u b=0.00356(+1)$ \\
$V c d=0.22527(+5)$ & $V c s=0.97344(1)$ & $V c b=0.0413(-1)$ \\
$V t d=0.00886(0)$ & $V t s=0.0405(0)$ & $V t b=0.99914(0)$ \\
\hline
\end{tabular}

The matrix normalization holds at better than $10^{-5}$. 
Note that removing the coefficients $1 / 7$ and $1 / 14$ in (21) leaves all Vij within $20 \%$ of the uncertainty range. Importantly, the appropriate coefficient might not be $1 / 137 \approx \alpha$ and $1 / 14 \approx 3 \pi \alpha$ but similar to the corrections used for $\theta_{\mathrm{W}}(12.1)$ and $\theta_{\mathrm{H}}(12.2)$.

Now out of the SM parameters related to quarks, and assuming $A_{S}$ is related to $\alpha_{S}$, we miss only the QCD vacuum angle which is $\sim 0$ but the overall picture is coherent with a single field; hence this parameter might not exist and be an excess of generality of the QCD theory. The same remark obviously applies to the standard CKM parameterization.

The Table 7 summarizes the main coefficients (without corrections) and mixings from T, B, C, and S.

Table 7. Mixing and main coefficients

\begin{tabular}{lll}
\hline$T / B, C / S \leftrightarrow T / C, B / S$ & $T / B, C / S \leftrightarrow S$ & $T / B, C / S \leftrightarrow B\left(1 / \pi \times 137^{2}\right)$ \\
$C \leftrightarrow T / C, B / S$ & $C \leftrightarrow S$ & $C \leftrightarrow B(1 / 2 \pi)$ \\
$T \leftrightarrow T / C, B / S\left(\pi / 137^{2}\right)$ & $T \leftrightarrow S(1 / 37)$ & $T \leftrightarrow B(1 / 3)$ \\
\hline
\end{tabular}

The Cabibbo angle links together two un-mixed states ( $c$ to $s$ ). It should then be possible to compute $V c s$ in a manner similar to the coefficients on the $t$ row and the $b$ column. Hence we define:

$$
\mathrm{fc}(\mathrm{q})=\mathrm{R}_{\mathrm{q}}^{2} /\left(\mathrm{R}_{\mathrm{s}}+\mathrm{R}_{\mathrm{d}}\right)^{2}
$$

And we empirically find:

$$
\begin{gathered}
\mathrm{Vcs}^{2}=1-\mathrm{fc}(\mathrm{s}) \times(3 / 4) \times(1-(1 / 137) \times(1-1 / 7))=(0.97344)^{2} \\
\mathrm{Vcd}^{2}=\left(\mathrm{fc}(\mathrm{d}) / \pi^{2}\right) \times(1-(1 / 4)(1 / \pi-1 / 37))=(0.22522)^{2}
\end{gathered}
$$

Using those numbers, the full matrix can be completed without the use of the Cabibbo angle, with similar precision as Table 6. A similar exercise with $V c s$ and $V u s$ is redundant but also gives good results. The interest of this approach is homogeneity in method, but also that the same numbers appear in the correction terms (even though it becomes a little far-fetched). It is numerically equivalent to the Cabibbo angle, but the latter seems physically more elegant and complete.

\subsubsection{Picturing the Quarks Field}

The Figures 3 shows how quarks are produced on rings 37 and 61 from T, B, C, S. (Each quark is represented by a circle that symbolizes 3 color states and spans over two hexagons.) Essentially, we find that only two quarks are mixes of the four others. This figure is, on purpose, reminiscent of the dissymmetry of matter and anti-matter; it suggests that the mixing dissymmetry effects exceed those expected with the standard model, and the dissymmetry does not require a different amount of positive and negative fundamental objects $(\mathrm{T}, \mathrm{B}, \mathrm{C}, \mathrm{S})$ to be created.
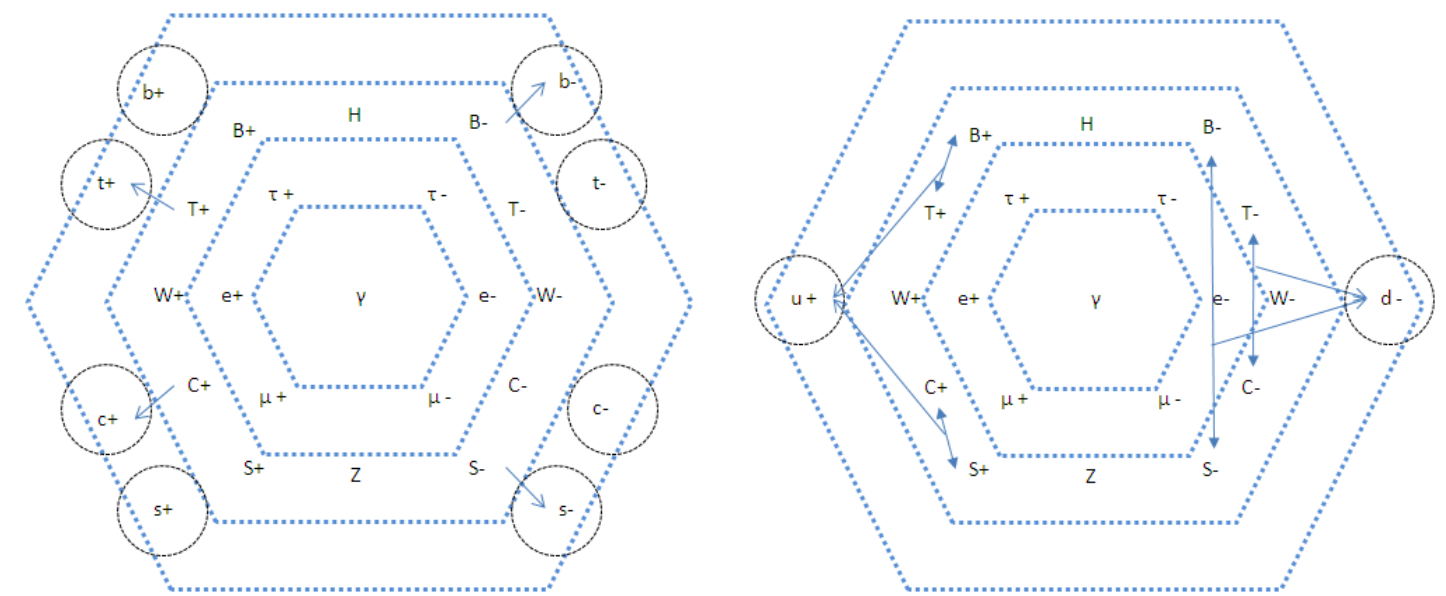

Figure 3. No mixing for $t, b, c$, and $s$ quarks (left). Mixing to the $u$ and $d$ quarks (right). 


\subsection{The Pontecorvo-Maki-Nakagawa-Sakata (PMNS) matrix}

Now let us see how the quarks mixing logic can be extended to neutrinos. We will discuss the PMNS matrix square elements as shown in Table 7; it is computed from the Particles Data Group (2014) ignoring the CP violating phase.

Table 8. The squared PMNS matrix. (Uncertainty is in the range $1-2 \%$.)

\begin{tabular}{lll}
\hline$U e_{1}{ }^{2}=0.6818$ & $U e_{2}{ }^{2}=0.2955$ & $U e_{3}{ }^{2}=0.0227$ \\
$U \mu_{1}{ }^{2}=0.3077$ & $U \mu_{2}{ }^{2}=0.6906$ & $U \mu_{3}{ }^{2}=0.0017$ \\
$U \tau_{1}{ }^{2}=0.0105$ & $U \tau_{2}{ }^{2}=0.0139$ & $U \tau_{3}{ }^{2}=0.9756$ \\
\hline
\end{tabular}

We first notice a pattern similar to the CKM matrix where most of the mixing is in the up-left corner.

$$
\begin{aligned}
\cos ^{-1}\left(U_{\mathrm{e} 1}\right) & =34.34^{\circ} \\
\sin ^{-1}\left(U_{\mathrm{e} 2}\right) & =32.93^{\circ} \\
\sin ^{-1}\left(U_{\mu 1}\right) & =33.69^{\circ} \\
\cos ^{-1}\left(U_{\mu 2}\right) & =33.80^{\circ}
\end{aligned}
$$

The difference is small compared to the matrix elements precision, and denotes a large almost equal mixing in electrons and muon neutrinos. Then we guess mixes and correction similar to the CKM for this part of the matrix. The mixing angle should be the arc-tangent of a ratio of lengths products; its resonance must be 1 and neutrinos are electrically neutral. Mixes are assumed from the same objects as the $u$ and $d$ quarks; then the resonances and electric neutrality leave no choice since $\mathrm{N}(\mathrm{t}) \mathrm{N}(\mathrm{s}) / \mathrm{N}(\mathrm{b}) \mathrm{N}(\mathrm{c})=1$ is minimal; now compute:

$$
\tan ^{-1}\left(\frac{\mathrm{R}_{\mathrm{t}} \mathrm{R}_{\mathrm{s}}}{\mathrm{R}_{\mathrm{b}} \mathrm{R}_{\mathrm{c}}}\right)=33.6^{\circ}
$$

which agrees with (22); but it works at the opposite of quarks mixing where a product of resonances gives a ratio of lengths; in (23), it corresponds to a product. This point is of high importance, firstly as it will be systematic in this section, and secondly as it implies the existence of an unexpected form of symmetry.

Now empirical corrections come in close agreement with (22); as follows:

$$
\begin{aligned}
& \tan ^{-1}\left(\frac{\mathrm{R}_{\mathrm{t}} \mathrm{R}_{\mathrm{s}}}{\mathrm{R}_{\mathrm{b}} \mathrm{R}_{\mathrm{c}}} \times\left(1+\frac{4}{137}\right)\right)=34.36^{\circ} \rightarrow \mathrm{U}_{\mathrm{e} 1} \\
& \tan ^{-1}\left(\frac{\mathrm{R}_{\mathrm{t}} \mathrm{R}_{\mathrm{s}}}{\mathrm{R}_{\mathrm{b}} \mathrm{R}_{\mathrm{c}}} \times\left(1-\frac{7}{274}\right)\right)=32.92^{\circ} \rightarrow \mathrm{U}_{\mathrm{e} 2} \\
& \tan ^{-1}\left(\frac{\mathrm{R}_{\mathrm{t}} \mathrm{R}_{\mathrm{s}}}{\mathrm{R}_{\mathrm{b}} \mathrm{R}_{\mathrm{c}}} \times\left(1+\frac{1}{274}\right)\right)=33.70^{\circ} \rightarrow \mathrm{U}_{\mu 1} \\
& \tan ^{-1}\left(\frac{\mathrm{R}_{\mathrm{t}} \mathrm{R}_{\mathrm{s}}}{\mathrm{R}_{\mathrm{b}} \mathrm{R}_{\mathrm{c}}} \times\left(1+\frac{1}{137}\right)\right)=33.79^{\circ} \rightarrow \mathrm{U}_{\mu 2}
\end{aligned}
$$

In the CKM matrix the T has a special role that should also be in the PMNS matrix. But $(23-24)$ include the product $R_{s} R_{t}$. Then the $S$ inherits of an anti-special role with respect to the $T$ (where $B$ and $C$ are not affected). On this basis, we easily find role-symmetry agreeing solutions giving the squared matrix elements. Bottom row:

$$
\begin{gathered}
1-\frac{\mathrm{R}_{\mathrm{t}}}{2\left(\mathrm{R}_{\mathrm{b}}+\mathrm{R}_{\mathrm{c}}+\mathrm{R}_{\mathrm{s}}\right)}=0.97541=\mathrm{U}_{\tau 3}^{2} \\
\frac{\mathrm{R}_{\mathrm{c}}}{42 \mathrm{R}_{\mathrm{s}}}=0.01046=\mathrm{U}_{\tau 1}^{2} \\
\frac{\mathrm{R}_{\mathrm{b}}}{21 \mathrm{R}_{\mathrm{s}}}=0.01389=\mathrm{U}_{\tau 2}^{2}
\end{gathered}
$$

The equation (25.1) shows that the product of resonances is not symmetrical (and can be written in many manners); its form is similar to the function $\mathrm{ft}(\mathrm{q})$ in (16.1) but based on reversed lengths- versus squared lengths or potentials. 
The right-hand column shows a direct symmetry with $(25.2-25.3)$ :

$$
\begin{gathered}
\left(\frac{\mathrm{R}_{\mathrm{c}}}{42 \mathrm{R}_{\mathrm{s}}}+\frac{\mathrm{R}_{\mathrm{b}}}{21 \mathrm{R}_{\mathrm{s}}}\right)\left(1-\frac{1}{14}\right)=0.02261=\mathrm{U}_{\mathrm{e} 3}^{2} \\
\left(\frac{\mathrm{R}_{\mathrm{c}}}{42 \mathrm{R}_{\mathrm{s}}}+\frac{\mathrm{R}_{\mathrm{b}}}{21 \mathrm{R}_{\mathrm{s}}}\right)\left(\frac{1}{14}\right)=0.001738=\mathrm{U}_{\mu 3}^{2}
\end{gathered}
$$

Interestingly, the coefficients 21 and 42 are multiples of 7 and the products NP of the $s$ and $c$ respectively; we also notice the recurrence of 7 which is the charged leptons ring and the $Z^{0}$ boson's K. Once again, the correction terms $1 / 137 \approx \alpha$ and $1 / 14 \approx 3 \pi \alpha$ may actually be the same as in $(12.1-12.2)$.

Using (24 - 25) gives appropriate results and the squared matrix normalization error is $5.410^{-4}$. The absolute error on the individual squared matrix elements with respect to Table 8 is lesser than $410^{-4}$ as shown in Table 9 .

Table 9. Computed PNMS squared matrix.

\begin{tabular}{lll}
$U e_{1}{ }^{2}=0.6814(-4)$ & $U e_{2}{ }^{2}=0.2954(-1)$ & $U e_{3}{ }^{2}=0.0226(-1)$ \\
$U \mu_{1}{ }^{2}=0.3078(1)$ & $U \mu_{2}{ }^{2}=0.6907(1)$ & $U \mu_{3}{ }^{2}=0.0017(0)$ \\
$U \tau_{1}{ }^{2}=0.0105(0)$ & $U \tau_{2}{ }^{2}=0.0139(0)$ & $U \tau_{3}{ }^{2}=0.9754(-2)$ \\
\hline
\end{tabular}

Compared to quarks, the mixing mechanism is different but based on the same lengths and objects; the numerical results are important evidences and it shows that the inferred core field is relevant. Moreover the same dissymmetry seems to exist as for quarks since we find the same corrections terms 1/14 in (25) and 1/137 in (24).

We shall not venture discuss neutrinos mass (Majorna or not), since mixing and oscillation appear related to an inversion of geometry or, as we shall discuss later, an inversion of dimensionality: For the neutrinos matrix, $\mathrm{N}(\mathrm{t}) \mathrm{N}(\mathrm{s}) / \mathrm{N}(\mathrm{b}) \mathrm{N}(\mathrm{c})$ gives the ratio $R_{t} R_{s} / R_{b} R_{c}$ where for quarks $N(t) / N(b)$ gives the product $R_{t} R_{b}$. A Majorna neutrino would leave room for a new particle (and matrix) in the sum to 61; a standard neutrino would imply that the mass (and possibly gravitational) interaction comes from a specific unknown neutrinos interaction.

Taking the inversion into account, the Figure 4 pictures neutrinos mix from ring 19; this is logically the electron and muon cases (with opposite inversions). The tau neutrino uses the same objects, but according to (25.1) it should be organized differently with identical roles for B, C, S, and a different role for T.

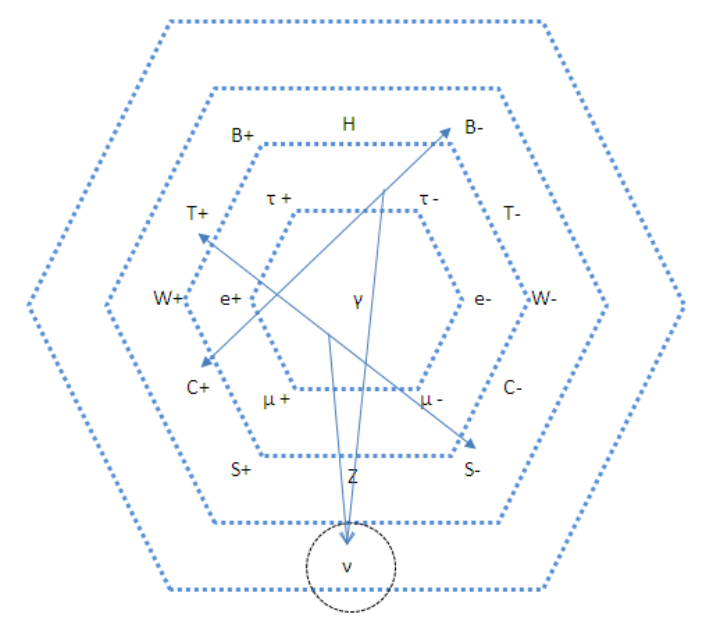

Figure 4. Neutrinos mixing - (logically the electron and muon neutrinos cases)

\subsection{Gluons}

Eight gluons must replace the eight objects and anti-objects (T, B, C, S) in the sum 61 as they are already accounted for as quarks. They should be defined with resonances $=1$ from $\mathrm{T}, \mathrm{B}, \mathrm{C}$, and $\mathrm{S}$, with the missing mixes, even though this is probably not complete since gluons and anti-gluons are pictured as the same objects appearing oppositely to quarks and anti-quarks (plane geometry comes short); that is $\mathrm{T}+\mathrm{T}-, \mathrm{T}-\mathrm{T}+, \mathrm{B}+\mathrm{B}-$, etc... 
This is presented in Figure 5, which is incomplete since a geometrical inversion should also be there (e.g. $N(t) / N(t)$ $\rightarrow \mathrm{R}_{\mathrm{t}} / \mathrm{R}_{\mathrm{t}}=1 \sim$ constant coupling).

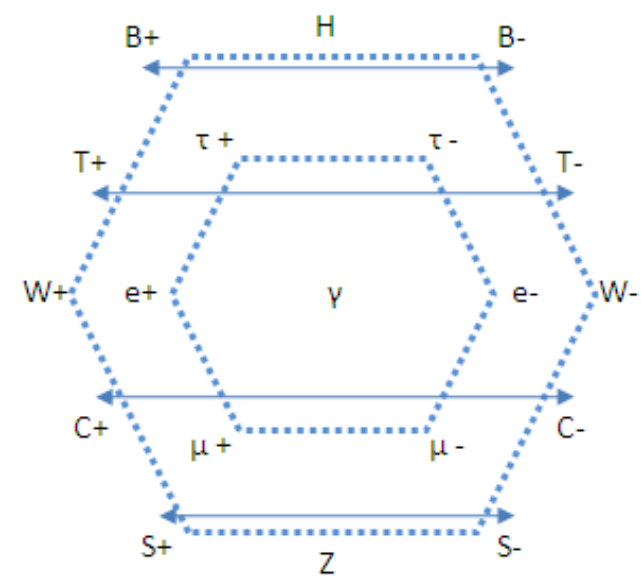

Figure 5. Gluons

\section{Definite Naturalness}

\subsection{Status}

The Table 10 shows the dependence of the SM (free) parameters on the results in this paper or the references.

Table 10. List of SM parameters and their status

\begin{tabular}{ccc}
\hline SM parameter & Compute from & Status \\
Leptons $\alpha$ Quarks Masses & Resonances & Theory \\
Higgs boson mass & Resonances & Theory \\
Higgs field vev & Bosons masses & Theory \\
$g 1, g 2$ & Alpha, Weakbosons masses & Theory \\
CKM and PMNS Matrices & $R_{q}(t, b, c, s)$ & Evidence \\
$g 3$ & Alpha_S $\left(D_{e}, \alpha\right)$ & Conjecture \\
QCD vacuum phase & Inexistent? & Conjecture \\
\hline
\end{tabular}

The next table shows a short list of open SM questions which are answered in this theory or logically conjectured.

Table 11. Short list of important open points in the SM

\begin{tabular}{ccc}
\hline SM open point & Solution & Status \\
Parameters origin? & Yes & Theory and Evidences \\
Fourth generation? & Yes & Prediction: None \\
EW and Gravitation? & $\theta_{H}$ & Conjecture \\
Naturalness? & Yes & $>80 \%$ \\
\hline
\end{tabular}

Note that the absence of $4^{\text {th }}$ generation (Consiglio, 2014b) is general and comes from opposite limits of the equation (1) when applied to leptons $(\mathrm{K} \mathrm{D}<1 / \mathrm{NP})$ and quarks $\left(\mathrm{m}_{\mathrm{q}}>0\right.$, or $\left.\mathrm{R}_{\mathrm{q}}>0\right)$.

We emphasize that our theory comes from a unique idea (composite currents) and a single equation from which every result presented in this paper follows. Essentially, we showed that the resonance mechanism and equation 
are coherent with all SM free parameters except the neutrinos CP violating phases which is out of reach at present, and the QCD vacuum phase and $\alpha_{\mathrm{S}}$, for which we raise the following hypothesis: The strong force coupling, at $\mathrm{M}_{\mathrm{Z}}$, is given by (2.3); the rationale for the scale $\mathrm{M}_{\mathrm{Z}}$ is that all masses are given by the inferred "absorber" which looks like a double-neutrino - hence a single interaction scale. Since the theory is consistent with a single field, we suggest that the QCD violating phase is inexistent and results from an excess of generality.

\subsection{Why Naturalness?}

The opposite question "why no naturalness" does not really need an answer; it is either un-natural and we can find many un-provable reasons (at first scary parameters) but a definite proof seems unlikely, or naturalness exists and requires progress. We possibly found "how" the universe is natural, but for a complete answer the question is also "why" since naturalness cannot be random. Our answer relates to self-encoding and self-coherence as both imply mathematical limitations or laws.

Firstly, the structure we find shows that the field is self-encoded or self-defined. We mean that all characteristics are structural as we find a figure defining all states where all parameters seem to be included; it should also include the system of co-ordinates - since interaction is an essential part of the "code".

Lorentz transformation shows that for $\mathrm{v}>\mathrm{c}$, time becomes space and conversely (reference in Consiglio, 2014a) but then this is the mirror used in the reasoning to equation (1) and it is a dimensional inversion. In this view it is logical that in field theory $\alpha_{S}\left(\sim D_{e} / \alpha+D_{e} \sqrt{ } 3\right)$ runs oppositely to $\alpha$ as $D_{e}$ and $\alpha$ come in opposite positions in the equation (2.3); they can be seen to "run alike" in reversed dimensions. Incidentally, we must recall that the first task of quantum mechanics is to remove the de Broglie wave phase velocity from the concept and equations.

With respect to dimensional inversion, de Broglie's $\mathrm{v} \rightarrow \mathrm{c}^{2} / \mathrm{v}$ can be read $\mathrm{r} \rightarrow 1 / \mathrm{r}$ and $\mathrm{t} \rightarrow 1 / \mathrm{t}$; accordingly, we compute from (14) a reversed pseudo-norm that speaks for itself:

$$
\gamma^{-1}=\sqrt{(8+2) \times 137-\frac{1}{137^{2}}-\frac{1}{\pi^{2}}}=37.01214
$$

...since 37 is the centered hexagonal number following 7 and 19 , and also the first mixing ring. The resonance of the top quark $(\mathrm{N}=38)$ is the only one exceeding this number - and as we know the top is special, firstly in mass and observations but also for its role in the above analysis of the CKM and PMNS matrices. Moreover we use lengths to compute the CKM matrix and lengths and reverse lengths for the PMNS matrix; similarly, the Cabibbo angle computed in (20) is coherent with products of lengths giving ratios of resonances. At the opposite in (23) the products come as if space and time were inverted for half of the mixing objects. Next, the bosons' $\mathrm{K}$ and quarks' $\mathrm{N}$ are equal and agree with an inversion of geometry. Last, the distances $\mathrm{D}_{\mathrm{e}}(2.1)$ and D' (13) can easily be evaluated as pseudo-norms of the same form as $\alpha(14)$, where the core field integral numbers appear (with no artifact); the following expressions hold at better than $10^{-9}$ (relative error):

$$
\begin{gathered}
\mathrm{D}_{\mathrm{e}}^{-1}=\sqrt{((7-3) \times(274+19))^{2}+(3+3+1) \pi^{2}-\frac{19 \pi}{19-1}} \\
\mathrm{D}^{\prime-1}=\sqrt{((19-3) \times(274+3))^{2}+2 \times(274+19+1) \pi^{2}-\frac{3}{3-1}}
\end{gathered}
$$

Hence, it looks like all results fit with an inversion of geometry or dimensionality, which (as far as we know) is unknown to existing theories but can be guessed already from de Broglie's thesis and special relativity.

Secondly, there should be a mathematical law limiting symmetries, which in turn must agree with or imply the hexagonal structure. Action implies reaction and the geometry of action/reaction is symmetry. This is always defined with sums and products, whatever the mathematical method used.

The limiting mathematical law that makes sense with respect to the SM in its present state is Hurwitz theorem (Dickson, 1919). It shows that only four alternative algebras exist, and three are isomorphic to the SM symmetry groups: Complex numbers $\mathrm{C} \sim \mathrm{U}(1)$, Quaternions $\mathrm{H} \sim \mathrm{SU}(2)$, and Octonions $\mathrm{O} \sim \mathrm{SU}(3)$; plus Real numbers $\mathrm{R}$.

Alternative algebra is defined by $(\mathrm{x} \mathrm{x}) \mathrm{y}=\mathrm{x}(\mathrm{x} \mathrm{y})$. We see in $(\mathrm{x} \mathrm{x})$ the self-interaction of a charge $\mathrm{x}$, and the other products are interaction, including $(\mathrm{x} \mathrm{x}) \leftrightarrow \mathrm{y}$ and $(\mathrm{x} \leftrightarrow \mathrm{y})$. This equality is simply the action of the charge $\mathrm{y}$ on the self-interaction of the charge $\mathrm{x}$, equal to the action on the charge $\mathrm{x}$ of the interaction of the charges $\mathrm{x}$ and $\mathrm{y}$. It links interaction and self-interaction, and it is trivial that the existence of a law of this type is needed in absorber theory. It is firstly a law of coherence and conservation. But the physical existence of self-interaction as the product (x x) indicates that particles are extended structures, firstly because self-interacting points do not make physical sense. 
Hence, considering Hurwitz theorem as a symmetry-limiting factor implies that the particle energy is an extended structure, like the equation (1) does.

Finally, and reversing the logic: A self-coherent universe that can be modeled with sums and products of charges and where particles are non-punctual structures is limited to $\mathrm{U}(1), \mathrm{SU}(2)$, and $\mathrm{SU}(3)$; it looks like the one we know.

\section{Conclusion}

Here we claim that the field is natural. Overall, we showed that at least 19 out of 23 SM parameters are coherent with each other, with the mass equation, and with the inferred field structure; one again, we emphasize that this result originates in a single idea and equation.

Firstly, we showed that the elementary particles mass spectrum is coherent and can be modeled; it results in a coherent and predictive theory. The very possibility that particles mass is structured is barred in field theory. We argue that this approach is incomplete as far as mass is concerned, and then also interaction.

Secondly, we showed that the Higgs boson seems much more important than suggested by the SM as it appears to call for more unification instead of a new separate field. We argue that the main gap to unification is that massive particles are composite of a charged current quantum.

Thirdly, we showed that the CKM and PMNS matrices come in a direct manner from the radiuses $\mathrm{R}_{\mathrm{q}}$ from which we compute the quarks masses. Hence we argue that the mass-resonance equation and its leading concept of charged currents are physical, and then key to model and understand the field.

\section{Addendum}

The manner we read the thermodynamics (the relation $P V=K_{B} T=\mu c^{2}=$ constant) in the equation (1) is not trivial; it is even somehow counter-intuitive since the volume $\mathrm{V}$ is the same as in the reasoning to the equation. On this basis, the equation (1) reads:

$$
m c^{2}=K_{B} T+X c^{2} /(1 / N P+K D)^{3}
$$

where the temperature $\mathrm{T}$ corresponding to $\mu$ is constant and the right-hand term is interaction (the absorber). Now imagine the particle as a ball (the cage) with a single gas molecule inside. When the interaction splits, for instance when a muon decays to a neutrino and an electron, the radius of the electron ball $R_{e}=\left(1 / 4+2 D_{e}\right)$ is larger than that of a muon $R_{\mu}=\left(1 / 25+3 D_{e}\right) ; K_{B} T$ is constant and only interaction changes. The molecule has a constant energy, but the compression of the ball depends on the inverse of the ball's volume. Now, of course, we can reverse the ball like a glove and we get an inverted picture. It is not obvious to understand which one should be preferred if any, and geometrical or dimensional inversion suggests that both are appropriate.

On the other hand, the constancy of $\mu=K_{B} T$ is rather stunning (in particular with respect to the toy model); but it was shown consistent since the ratio $\mathrm{X} / \mu$ is the basis enabling to compute the leptons magnetic moment anomaly (Consiglio, 2015). It turns-out from this calculus that $\mu$ is just the interaction of the time-currents together; and leptons are a 4D rotation of currents around a plane defined by the magnetic moment and the time axis.

In the example above (electron and muon), it is natural to identify the rotation rate with the particle energy and frequency, that is $E=h v$. Now both particles have the same intrinsic angular momentum $\mathrm{h} / 2$; but $\mu$ is constant and then the classical rotation radius is in reverse proportion of the particle frequency: It looks like the Compton wavelength and the difficulty, if any, is to imagine its dependence on a volume.

\section{References}

De Broglie, L. (1924) Recherches sur la théorie des quanta. Annales de Physique - 10e sérié - Tome III Janvier-Février 1925.

Cramer, J. (1986). The transactional interpretation of quantum mechanics. Rev. Mod. Phys., 58(3). http://dx.doi.org/10.1103/RevModPhys.58.647

Consiglio, J. (2014a) Below the Standard Model? Applied Physics Research, 6(2), 19-27. http://dx.doi.org/10.5539/apr.v6n2p19

Consiglio. (2014b). On the Field Below SM, Analysis and Predictions. Applied Physics Research, 6(5), 1-17. http://dx.doi.org/10.5539/apr.v6n5p1

Consiglio, J. (2015) Hacking the Fine Structure Constant in Leptons Geometry. Applied Physics Research, 1(7), 30-46. http://dx.doi.org/10.5539/apr.v716p30

Dickson, L. E. (1919). On Quaternions and Their Generalization and the History of the Eight Square Theorem. Ann. Math., 20, 155. http://dx.doi.org/10.2307/1967865 
Poincaré, H. (1903) Sur la dynamique de l'électron. Rendiconti del Circolo Matematico di Palermo, 21(1906), 129-176. http://dx.doi.org/10.1007/bf03013466

Wheeler, J. A., \& Feynman, R. P. (1945). Interaction with the Absorber as the Mechanism of Radiation. Reviews of Modern Physics, 17(2-3), 157-161. http://dx.doi.org/10.1103/RevModPhys.17.157

Wheeler, J. A., \& Feynman, R. P. (1949). Classical Electrodynamics in Terms of Direct Interparticle Action. Reviews of Modern Physics, 21(3), 425-433. http://dx.doi.org/10.1103/RevModPhys.21.425

ATLAS collaboration. (2014). Measurement of the Higgs boson mass from the $\mathrm{H} \rightarrow \gamma \gamma$ and $\mathrm{H} \rightarrow \mathrm{ZZ} \rightarrow 41$ channels in pp collisions at center-of-mass energies of 7 and $8 \mathrm{TeV}$ with the ATLAS detector". Phys. Rev. D (American Physical Society), 90(5), 052004. http://dx.doi.org/10.1103/PhysRevD.90.052004

CMS collaboration. (2014a). Precise determination of the mass of the Higgs boson and studies of the compatibility of its couplings with the standard model (Technical report). CMS-PAS-HIG-14-009. CERN. Retrieved from https://cds.cern.ch/record/1728249? ln=en

ATLAS and CMS Collaborations. (2015). Combined Measurement of the Higgs Boson Mass in pp Collisions at $\sqrt{ }_{\mathrm{s}}=7$ and $8 \mathrm{TeV}$ with the ATLAS and CMS Experiments.

The ATLAS, CDF, CMS and D0 Collaborations. (2013). First combination of Tevatron and LHC measurements of the top-quark mass. arXiv, 1403, 4427

The CMS Collaboration. (2014b). Measurement of the top-quark mass in top-quark pair events with lepton+jets final states in pp collisions at sqrt(s)=8 TeV. CMS Physics Analysis Summary TOP-14-001. Retrieved from https://cds.cern.ch/record/1690093?ln=en

\section{Copyrights}

Copyright for this article is retained by the author(s), with first publication rights granted to the journal.

This is an open-access article distributed under the terms and conditions of the Creative Commons Attribution license (http://creativecommons.org/licenses/by/3.0/). 